\title{
Guest Editorial: Embedded Multicore Systems and Applications
}

\author{
Jenq Kuen Lee • Albert Cohen • Roy Ju • Kuan-Ching Li
}

Published online: 31 January 2015

(C) Springer Science+Business Media New York 2015

Embedded systems with multi-core designs are becoming increasingly important for signal processing and multimedia applications. While embedded multi-core systems will look to play an important role ahead for application designs, many challenging problems remain to be solved. Applications, programming models, compilers, architecture designs, and software tools all need to contribute to the advance of embedded multi-core computing for signal processing and multimedia applications.

The special issue on "Embedded Multicore Systems and Applications" brings together a rich blend of theory and practice in embedded computer architecture, parallel algorithms, runtime systems, compilers, networking, and performance evaluation. After each submission was reviewed by at least 3 experts and associate editors, the guest editors decided to accept 6 papers.

The first paper, "An Adaptive Heterogeneous Runtime Framework for Irregular Applications" (10.1007/s11265014-0916-x), by Kao et al., is focused on managing the heterogeneous computing resources for the irregular

J. K. Lee

National Tsing Hua University, Hsinchu, Taiwan

e-mail: jklee@cs.nthu.edu.tw

\section{A. Cohen $(\bowtie)$}

École Normale Supérieure, Paris, France

e-mail: Albert.Cohen@inria.fr

R. Ju

MediaTek, San Francisco, CA, USA

e-mail: Roy.Ju@mediatek.com

K.-C. Li

Providence University, Taichung City, Taiwan

e-mail: kuancli@pu.edu.tw applications. This paper presents a phase guided dynamic work partitioning framework for efficient work dispatching on a heterogeneous system. Such an approach tries to resolve the control flow divergence and load imbalance issues in irregular applications. The paper evaluates a RayTracing application with the proposed method and shows significant performance gain.

There are three papers related to performance and power optimizations for multi-core embedded computing in this special issue. The paper titled "An Auto-tuning Assisted PowerAware Study of Iris Matching Algorithm on Intel's SCC" (10. 1007/s11265-014-0901-4), authored by Torres et al., employed an Intel Single-chip Cloud Computer (SCC) to investigate the power-aware computing and performance enhancement of an iris matching algorithm on many-core architectures. The authors study various metrics and analyzed the results with different system parameters, including performance, power, energy, energy delay product, and power per speedup. Finally, they propose an auto-tuning method for quickly approaching the optimal configuration of the SCC based on the targeted metric.

The paper "Compilers for Low Power with Design Patterns on Embedded Multicore Systems" (10.1007/ s11265-014-0917-9), by Lin et al., presents a set of power optimization schemes in compiler by exploiting the recurring patterns of embedded multicore applications. In this paper, the authors present a series of pattern-based pragmas to identify a specific parallel pattern for guiding compiler for power optimization. In their experiments, several multicore applications are evaluated with the proposed power optimization methods and have shown significant energy reduction.

The third paper in the category of performance and power optimizations, "Performance and Energy Evaluation of 
Different Multi-Threading Interfaces in Embedded and General Purpose Systems" (10.1007/s11265-014-0925-9), authored by Lorenzon et al., presents a detailed study on how the multi-threading programming interfaces influence on performance, energy, and energy delay product in embedded and general systems. Three major APIs, OpenMP, MPI, and Pthread are evaluated with 8 benchmarks that contain both CPU- and memory-bound programs.

The paper entitled "Multi-core DSP-based Vector Set Bits Counter/Comparators" (10.1007/s11265-014-0915-y), authored by Sklyarov et al., presents a hardware accelerator solution for fast counting and comparing vector bits which are also known as Hamming weights and Hamming distance. The proposed solution is based on multicore FPGA-based accelerators. Detailed hard ware specification is given for analyzing and comparing with best known hardware/software solutions. They evaluate the proposed design and show significant speed-up while comparing to the known hardware/ software alternatives.

The last paper in this special issue, titled "RCSoS: An IEC 61508 Compatible Server Model for Reliable Communication" (10.1007/s11265-014-0914-z), authored by Zhou et al., proposes a server model that utilizes the synergistic processor unit (SPU) in Cell/B.E. platform for reliable communication. Their building process confirms IEC 61508, which is an international standard that standardizes the functional safety for safetyrelated systems. The experimental results show the sta ble and acceptable performance of the proposed server model.

The above six papers in this special issue cover different aspects of multi-core embedded computing and applications for signal processing. These papers provide frontier information related to the embedded computer architecture, parallel algorithms, runtime systems, compilers, networking, and performance evaluation in the embedded multi-core computing for signal processing systems.

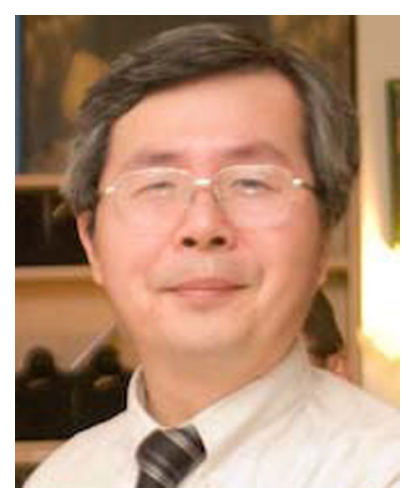

Jenq Kuen Lee received the B.S. degree in computer science from National Taiwan University in 1984. He received the MS and $\mathrm{PhD}$ degrees in 1991 and 1992, respectively,in computer science from Indiana University. He is now a professor in the Department of Computer Science at National Tsing-Hua University, Taiwan, where he joined the Department in 1992. He was a key member of the team who developed the first version of the $\mathrm{pC}++$ language and SIGMA system while at Indiana University. He was also a recipient of the most original paper award in ICPP '97 with the paper entitled "Data Distribution Analysis and Optimization for Pointer-Based Distributed Programs". In 2005, he received Taiwan MOEA funding to lead a research team to develop compilers for PAC VLIW DSP processors with distributed register files by collaborating with ITRI STC. He is also a recipient of Google Research Award (Mountain View), 2009. He was also a director for Taiwan MOE ESW (embedded system software) consortium 2008-2013. In 2010, he received a Taiwan MOEA economic contribution award (Deep Plow Award) for his contribution in embedded compiler research. His research interests are in optimizing compilers, embedded compilers, and computer architectures.

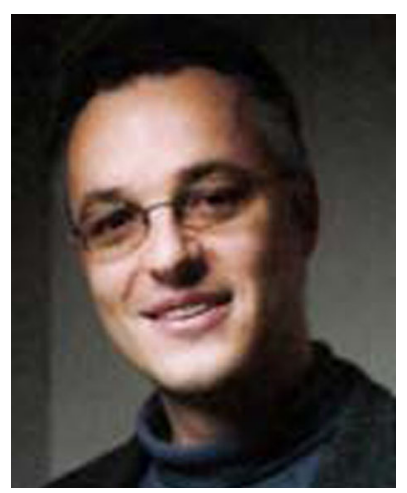

Albert Cohen is a senior research scientist at INRIA and a part-time associate professor at École Polytechnique. He graduated from École Normale Supérieure de Lyon, and received his $\mathrm{PhD}$ from the University of Versailles in 1999 (awarded two national prizes). He has been a visiting scholar at the University of Illinois and an invited professor at Philips Research, both for 6 months. Albert Cohen works on parallelizing and optimizing compilers, parallel programming, and synchronous programming for embedded systems. He has been the general chair of major conferences, including PLDI, PPoPP, HiPEAC, he is a member of the editorial board of ACM TACO, IJPP and IJES, and as the program chair of CC, ARCS, APPT. He coauthored more than 120 peer-reviewed papers, and has been the advisor for $22 \mathrm{PhD}$ theses. Several research projects initiated by Albert Cohen resulted in effective transfer to production compilers. 


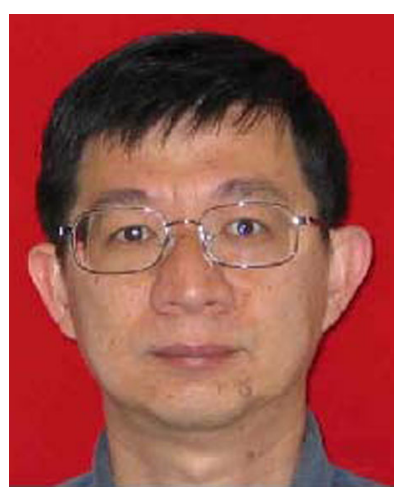

Roy D.C. Ju is a Sr. Technical Director at MediaTek and driving its Heterogeneous Computing roadmap and developments. He was with Advanced Micro Devices from 2006 to 2013. He was the compiler architect for the Heterogeneous System Architecture (HSA) project, and was promoted to AMD Senior Fellow in 2013. From 1999 to 2005 , he was a senior researcher and the manager of Compiler Technology group at the Programming System Lab in the Microprocessor Technology Labs, Intel Corp. He had been the architect of an IA-64 open source research compiler (Open Research Compiler), which provided an infrastructure for compiler and architecture research on IA-64 to the research and open source communities. He was with the Hewlett-Packard Company from 1994 to 1999, where he was a project lead in designing and developing an optimizing compiler for IA-64. He worked at IBM from 1992 to 1994 in developing a then state-of-the-art Fortran 90 optimizing compiler. His primary research interests include compiler optimizations, optimization for memory hierarchy, program analysis, computer architecture, multi-core systems, and parallel processing. He received his B.S. in Electrical Engineering from National Taiwan University in 1984. He received his M.S. and Ph.D. in Electrical and Computer Engineering from the University of Texas at Austin in 1988 and 1992, respectively.

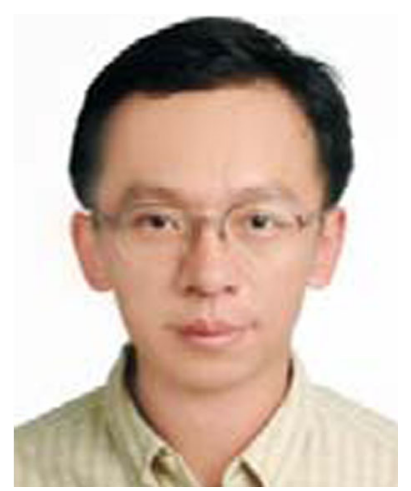

Kuan-Ching Li is a Professor in the Department of Computer Science and Information Engineering at the Providence University, Taiwan. He was a department chair in 2009, the Special Assistant to the University President since 2010, and appointed as ViceDean for Office of International and Cross-Strait Affairs in this same university since 2014. Dr. $\mathrm{Li}$ is recipient of several awards that includes Nvidia, Ministry of Education (MOE)/Taiwan and Ministry of Science and Technology (MOST)/Taiwan, as also recipient of guest professorships from universities in China. He serves/has served on the chairmanship positions of several conferences and workshops, and has organized numerous conferences related to high-performance computing and computational science \& engineering. Dr. Li is the Editor-in-Chief of technical publications International Journal of Computational Science and Engineering (IJCSE), International Journal of Embedded Systems (IJES) and International Journal of High Performance Computing and Networking (IJHPCN), all published by Inderscience, also serving a number of journal's editorial boards and guest editorship. His topics of interest include networked computing, GPU computing, parallel software design, and performance evaluation and benchmarking. Dr. Li is a Fellow of the IET and a senior member of the IEEE. 